Article

\title{
Best Approximation Results in Various Frameworks
}

\author{
Taoufik Sabar, Abdelhafid Bassou * and Mohamed Aamri
}

Laboratory of Algebra, Analysis and Applications (L3A), Departement of Mathematics and Computer Science, Faculty of Sciences Ben M'sik, Hassan II University of Casablanca, P.B 7955 Sidi Othmane, Casablanca, Morocco; sabarsaw@gmail.com (T.S.); aamrimohamed82@gmail.com (M.A.)

* Correspondence: hbassou@gmail.com

Received: 20 April 2019; Accepted: 16 May 2019; Published: 27 May 2019

check for updates

\begin{abstract}
We first provide a best proximity point result for quasi-noncyclic relatively nonexpansive mappings in the setting of dualistic partial metric spaces. Then, those spaces will be endowed with convexity and a result for a cyclic mapping will be obtained. Afterwards, we prove a best proximity point result for tricyclic mappings in the framework of the newly introduced extended partial $S_{b}$-metric spaces. In this way, we obtain extensions of some results in the literature.
\end{abstract}

Keywords: best proximity point; dualistic partial metric space; tricyclic mappings; extended partial $S_{b}$-metric space

\section{Introduction}

Whether a self mapping has fixed points or not is a problem that has been exhaustively studied ever since Banach stated his contraction principle. In the beginning of the current century, an issue of equivalent importance to that of the fixed point problem appeared: Let $T$ be a cyclic (resp. noncyclic) mapping on $A \cup B$ where $A$ and $B$ are nonempty subsets of a metric space $(X, d)$, that is, $T(A) \subseteq B$ and $T(B) \subseteq A$ resp. $T(A) \subseteq A$ and $T(B) \subseteq B)$. The equation $T x=x$ may not possess a soltution, in this case, we wish to determine an element (resp. a pair) which is as close to its image as possible, i.e., an element $x \in A \cup B$ such that $d(x, T x)=\operatorname{dist}(A, B)$ (resp. a pair $(x, y) \in A \times B$ of fixed points such that $d(x, y)=\operatorname{dist}(A, B)$ ). Such a point (resp. pair) is called a best proximity point (resp. pair). The problem of best approximation for cyclic and noncyclic mappings attracted a good many authors and many pertinent results were obtained in different frameworks [1-7].

In 2011, the notion of $P$-property was introduced in [8] and best proximity point results for weakly contractive non-self-mappings were obtained. Two years later, using the aforementioned property, Abkar and Gabaleh [9] proved that some existence and uniqueness results in best proximity point theory can be acquired from existing results in the fixed point theory. In the same year, Almeida, Karapinar and Sadarangani [10] showed that best proximity point results can be obtained from fixed point results using only the weaker condition of weak $P$-property. In 2016, Ref. [11] presented a new approach to best proximity point results by means of the so-called simulation functions.

In 2017, Sabar, Aamri and Bassou [12] introduced the class of tricyclic mappings and best proximity points thereof. Let $A, B$ and $C$ be nonempty subsets of a metric space $(X, d)$. A mapping $T: A \cup$ $B \cup C \longrightarrow A \cup B \cup C$ is said to be tricyclic if $T(A) \subseteq B, T(B) \subseteq C$ and $T(C) \subseteq A$, and a best proximity point for $T$ is an element $x \in A \cup B \cup C$ such that $D\left(x, T x, T^{2} x\right)=\operatorname{dist}(A, B, C)$ where $D(x, y, z)=d(x, y)+d(y, z)+d(z, x)$ and

$$
\operatorname{dist}(A, B, C)=\inf \{D(x, y, z): x \in A, y \in B \text { and } z \in C\} \text {. }
$$


This paper aims to establish best proximity point results for subclasses of cyclic, noncyclic and tricylic mappings in the framework of partial dualistic metric spaces and the lately introduced extended partial $S_{b}$-metric spaces [13].

\section{Best Proximity Point Results in Dualistic Partial Metric Spaces}

This section deals with cyclic and noncyclic mappings in dualistic partial metric spaces; these spaces were first introduced as follows.

Definition 1 ([14]). Let $X$ be a nonempty set. A function $\mathcal{D}: X \times X \longrightarrow \mathbb{R}$ is called a dualistic partial metric if

$\left(D_{1}\right) x=y$ if and only if $\mathcal{D}(x, x)=\mathcal{D}(y, y)=\mathcal{D}(x, y)$,

$\left(D_{2}\right) \mathcal{D}(x, x) \leq \mathcal{D}(x, y)$,

$\left(D_{3}\right) \mathcal{D}(x, y)=\mathcal{D}(y, x)$,

$\left(D_{4}\right) \mathcal{D}(x, y) \leq \mathcal{D}(x, z)+\mathcal{D}(z, y)-\mathcal{D}(z, z)$,

for all $x, y, z \in X$.

Complying with [14], $\mathcal{D}$ generates a $T_{0}$ topology on $X$, denoted by $\tau(\mathcal{D})$ in which the open balls are

$$
\left\{B_{\mathcal{D}}(x, \varepsilon): x \in X, \varepsilon>0\right\} \quad \text { where } B_{\mathcal{D}}(x, \varepsilon)=\{y \in X: \mathcal{D}(x, y)<\varepsilon+\mathcal{D}(x, x)\} .
$$

Now, we are able to introduce the notions of convergence and Cauchy sequences in the setting of dualistic partial metric spaces.

Definition 2 ([15]). A sequence $\left(x_{n}\right)$ in $(X, \mathcal{D})$ converges to a point $x$ if and only if $\mathcal{D}(x, x)=$ $\lim _{n \longrightarrow \infty} \mathcal{D}\left(x_{n}, x\right)$ and it is a Cauchy sequence if $\lim _{n} \longrightarrow \infty \mathcal{D}\left(x_{n}, x_{m}\right)$ exists and it is finite.

To present our results, we need to mention some basic concepts related to noncyclic mappings. In this section, unless stated otherwise, $A$ and $B$ are nonempty subsets of a dualistic partial metric space $(X, \mathcal{D})$ and $T: A \cup B \longrightarrow A \cup B$ is a noncyclic mapping:

$$
\begin{aligned}
F_{A}(T) & =\{x \in A: T x=x\} \text { and } F_{B}(T)=\{y \in B: T y=y\} \\
\operatorname{dist}(A, B) & =\inf \{\mathcal{D}(x, y): x \in A, y \in B\} \\
A_{0} & =\{x \in A: \mathcal{D}(x, y)=\operatorname{dist}(A, B) \text { for some } y \in B\} \\
B_{0} & =\{y \in B: \mathcal{D}(x, y)=\operatorname{dist}(A, B) \text { for some } x \in A\}
\end{aligned}
$$

Definition 3. The mapping $T$ is said to be relatively nonexpansive if

$$
\mathcal{D}(T x, T y) \leq \mathcal{D}(x, y) \text { for all } x \in A \text { and } y \in B .
$$

In addition, a pair $(x, y) \in A \times B$ is said to be a best proximity pair if

$$
x \in F_{A}(T), y \in F_{B}(T) \text { and } \mathcal{D}(x, y)=\operatorname{dist}(A, B) .
$$

In [16], Gabeleh and Otafudu introduced the class of quasi-noncyclic relatively nonexpansive mappings as follows.

Definition 4. Suppose $A_{0} \neq \varnothing$. The mapping $T$ is said to be quasi-noncyclic relatively nonexpansive mapping provided that $\left(F_{A_{0}}(T), F_{B_{0}}(T)\right) \neq \varnothing$ and, for all $(a, b) \in F_{A_{0}}(T) \times F_{B_{0}}(T)$, we have

$$
\left\{\begin{array}{l}
\mathcal{D}(T x, b) \leq \mathcal{D}(x, b) \text { for all } x \in A, \\
\mathcal{D}(a, T y) \leq \mathcal{D}(a, y) \text { for all } y \in B .
\end{array}\right.
$$


The class of quasi-noncyclic relatively nonexpansive mappings is not a subclass of noncyclic relatively nonexpansive mappings. To check that out and for more constructions on quasi-noncyclic relatively nonexpansive mappings, we refer the reader to [17,18].

Definition 5. $A$ is said to be approximatively compact with respect to $B$ if and only if every sequence $\left(x_{n}\right)$ in $A$ such that $\mathcal{D}\left(y, x_{n}\right) \longrightarrow \mathcal{D}(y, A)$ for some $y \in B$ has a convergent subsequence.

\section{Remark 1.}

- If $A$ is a compact set, then it is approximatively compact with respect to $B$.

- If $A \cap B \neq \varnothing$, then $A$ is approximatively compact with respect to $A \cap B$. Indeed, let $\left(x_{n}\right)$ in $A$ such that $\mathcal{D}\left(y, x_{n}\right) \longrightarrow \mathcal{D}(y, A)$ for some $y \in A \cap B$. Since $\mathcal{D}(y, y) \leq \mathcal{D}(y, x)$ for all $x \in X, \mathcal{D}(y, A)=\mathcal{D}(y, y)$ and that means $\left(x_{n}\right)$ converges to $y$.

Definition 6 ([19]). The pair $(A, B)$ is called sharp (resp. semi-sharp) proximal if and only if, for each $x$ in $A$ and $y$ in $B$, there exist a unique (resp. at most one) element $x^{\prime}$ in $B$ and a unique element $y^{\prime}$ in $A$ such that

$$
\mathcal{D}\left(x, x^{\prime}\right)=\mathcal{D}\left(y^{\prime}, y\right)=\operatorname{dist}(A, B)
$$

Now, we're entitled to state our first main result.

Theorem 1. Let $(X, \mathcal{D})$ be a dualistic partial metric space such that $\mathcal{D}$ is continuous and let $A, B$ be nonempty subsets of $X$ such that $A_{0} \neq \varnothing, B$ is approximatively compact with respect to $A$ and the pair $(A, B)$ is semi-sharp proximal. Then, each quasi-noncyclic relatively nonexpansive mapping defined on $A \cup B$ possesses a best proximity pair.

Proof. Let $\left(x_{n}\right)$ be a sequence of elements of $A_{0}$ which converges to some $x \in F_{A_{0}}(T)$. ( The fact that $F_{A_{0}}(T)$ is nonempty guarantees the existence of such a sequence ). Choose a point $y_{n}$ in $B_{0}$ such that

$$
\mathcal{D}\left(x_{n}, y_{n}\right)=\operatorname{dist}(A, B) \text { for all } n \in \mathbb{N} .
$$

Now, we get

$$
\begin{aligned}
\mathcal{D}\left(x, y_{n}\right) & \leq \mathcal{D}\left(x, x_{n}\right)+\mathcal{D}\left(x_{n}, y_{n}\right)-\mathcal{D}\left(x_{n}, x_{n}\right) \\
& =\mathcal{D}\left(x, x_{n}\right)+\operatorname{dist}(A, B)-\mathcal{D}\left(x_{n}, x_{n}\right) \\
& \leq \mathcal{D}\left(x, x_{n}\right)+\operatorname{dist}(x, B)-\mathcal{D}\left(x_{n}, x_{n}\right) .
\end{aligned}
$$

Taking into account that $\mathcal{D}$ is a continuous mapping on $X \times X$, we get

$$
\mathcal{D}\left(x_{n}, x_{n}\right) \longrightarrow \mathcal{D}(x, x) \text { as } n \longrightarrow \infty .
$$

Therefore, letting $n \longrightarrow \infty$, we obtain $\mathcal{D}\left(x, y_{n}\right) \longrightarrow \operatorname{dist}(x, B)$. The hypothesis that $B$ is approximatively compact with respect to $A$ implies the existence of a subsequence $\left(y_{n_{k}}\right)$ of $\left(y_{n}\right)$ and a $y \in B$ such that $y_{n_{k}} \longrightarrow y$ as $k \longrightarrow \infty$. Hence, $\operatorname{dist}(A, B)=\mathcal{D}\left(x_{n_{k}}, y_{n_{k}}\right) \longrightarrow \mathcal{D}(x, y)$, which means

$$
\mathcal{D}(x, y)=\operatorname{dist}(A, B) .
$$

Since $T$ is quasi-noncyclic relatively nonexpansive,

$$
\mathcal{D}(x, T y) \leq \mathcal{D}(x, y)=\operatorname{dist}(A, B) .
$$

Now, we use the assumption that the pair $(A, B)$ is semi-sharp proximal to conclude that $y$ is a fixed point and therefore $(x, y)$ is a best proximity pair. 
Example 1. Let $X=\mathbb{R}^{2}$ with the dualistic partial metric $\mathcal{D}\left((x, y),\left(x^{\prime}, y^{\prime}\right)\right)=\max \left\{x, x^{\prime}\right\}+$ $\max \left\{y, y^{\prime}\right\}$. Let $A=\{0\} \times[0, \infty)$ and $B=\{1\} \times[0, \infty)$. Then, $A_{0}=\{(0,0)\}$ and $\operatorname{dist}(A, B)=1$. Moreover, the pair $(A, B)$ is semi-sharp proximal. Let $T: A \cup B \longrightarrow A \cup B$ be a noncyclic mapping such that $T(0, x)=(0, x / 2)$ and $T(1, x)=(1, x / 2)$ for all $x \in[0, \infty)$. Clearly, $T$ is $a$ quasi-noncyclic relatively nonexpansive and its best proximity pair is $((0,0),(1,0))$.

As a special case of the previous theorem, we obtain the following result which was proven in [20].

Corollary 1. (Theorem 1 of [20]) Let $(X, d)$ be a complete metric space and $A, B$ be nonempty subsets of $X$ such that $A$ is closed and $A_{0} \neq \varnothing$. Suppose that $B$ is approximatively compact with respect to $A$ and that $T: A \cup B$ $\longrightarrow A \cup B$ is a quasi-noncyclic mapping such that $T \mid A$ is a contraction in the sense of Banach, $T\left(A_{0}\right) \subseteq A_{0}$ and the pair $(A, B)$ is semi-sharp proximal. Then, $T$ has a best proximity pair.

The notion of convexity in metric spaces was firstly introduced in [21] and the exact same notion can be given in dualistic partial metric spaces.

Definition 7. A mapping $W: X \times X \times[0,1] \longrightarrow X$ is said to be a convex structure on $X$ if, for each $(x, y) \in X \times X$ and $\lambda \in[0,1]$,

$$
\mathcal{D}(u, W(x, y, \lambda)) \leq \lambda \mathcal{D}(u, x)+(1-\lambda) \mathcal{D}(u, y) \text { for all } u \in X .
$$

In addition, $(X, \mathcal{D}, W)$ is said to be a convex dualistic partial metric space.

Definition 8. A subset $K$ of a convex dualistic partial metric space $(X, \mathcal{D}, W)$ is said to be convex if $W(x, y, \lambda) \in K$ for all $x, y \in K$ and $\lambda \in[0,1]$.

The following propositions are immediate.

Proposition 1 ([21]). Let $\left\{K_{\alpha}\right\}_{\alpha \in A}$ be a family of convex subsets of the convex dualistic partial metric space X; then, $\cap_{\alpha \in A} K_{\alpha}$ is also a convex subset of $X$.

Proposition 2. The closed ball centered at $a \in X$ with radius $r \in \mathbb{R}$ is a convex subset of $X$.

Proof. Let $x, y \in B(a, r)$ and $\lambda \in[0,1]$,

$$
\begin{aligned}
\mathcal{D}(a, W(x, y, \lambda)) & \leq \lambda \mathcal{D}(a, x)+(1-\lambda) \mathcal{D}(a, y) \\
& \leq \lambda(r+\mathcal{D}(a, a))+(1-\lambda)(r+\mathcal{D}(a, a)) \\
& \leq r+\mathcal{D}(a, a)
\end{aligned}
$$

In addition, this means that the closed ball is convex.

Definition 9. A convex dualistic partial metric space $(X, \mathcal{D}, W)$ is said to verify property $(C)$ if every bounded increasing net of nonempty, closed and convex subsets of $X$ is of nonempty intersection.

A weakly compact convex subset of a Banach space has property $(C)$ for instance. For more examples, we allude to [22].

Let $A$ and $B$ be nonempty subsets of a convex dualistic partial metric space $(X, \mathcal{D}, W)$. We set

$$
\begin{aligned}
& \delta(A, B)=\sup \{\mathcal{D}(x, y): x \in A \text { and } y \in B\} \\
& \delta_{(x)}(B)=\sup \{\mathcal{D}(x, y): y \in B\} \text { for all } x \in A
\end{aligned}
$$


By $\overline{c o n}(A)$, we denote the closed and convex hull of $A$ and it is defined by

$$
\overline{\operatorname{con}}(A)=\cap\{C: C \text { is a closed and convex subset of } X \text { such that } C \supseteq A\} .
$$

The following lemma is used in the proof of our second main result of this section.

Lemma 1. Let $(A, B)$ be a nonempty, bounded, closed, and convex pair in a convex dualistic partial metric space $(X, \mathcal{D}, W)$. Suppose that $T: A \cup B \rightarrow A \cup B$ is a cyclic mapping. If $X$ has the property $(C)$, then there exists a pair $\left(K_{1}, K_{2}\right) \subseteq(A, B)$ which is maximal with respect to being nonempty, closed and convex such that $T$ is cyclic on $K_{1} \cup K_{2}$. Furthermore,

$$
\overline{c o}\left(T\left(K_{1}\right)\right)=K_{2} \text { and } \overline{c o}\left(T\left(K_{2}\right)\right)=K_{1} .
$$

Proof. The set of all nonempty, closed, and convex pairs $(C, D) \subseteq(A, B)$ such that $T$ is cyclic on $C \cup D$ is partially ordered by reverse inclusion, i.e.,

$$
\left(C_{1}, D_{1}\right) \leq\left(C_{2}, D_{2}\right) \Longleftrightarrow\left(C_{2}, D_{2}\right) \subseteq\left(C_{1}, D_{1}\right) .
$$

For each increasing chain $\left\{\left(C_{\alpha}, D_{\alpha}\right)\right\}_{\alpha}$, we set $C:=\cap C_{\alpha}$ and $D:=\cap D_{\alpha}$. Since $X$ has the property $(C)$ and from the fact that every intersection of convex subsets is a convex subset, $(C, D)$ is a nonempty, closed and convex pair. In addition,

$$
T(C) \subseteq T\left(\cap C_{\alpha}\right) \subseteq \cap T\left(C_{\alpha}\right) \subseteq \cap D_{\alpha}=D .
$$

Similarly, $T(D) \subseteq C$, which means that $T$ is cyclic on $C \cup D$. Therefore, every increasing chain is bounded above and Zorn's Lemma assures the existence of the maximal pair $\left(K_{1}, K_{2}\right)$. Now, we note that the pair $\left(\overline{c o}\left(T\left(K_{2}\right)\right), \overline{c o}\left(T\left(K_{1}\right)\right)\right) \subseteq\left(K_{1}, K_{2}\right)$ is nonempty, closed and convex. We also have

$$
T\left(\overline{c o}\left(T\left(K_{2}\right)\right)\right) \subseteq T\left(K_{1}\right) \subseteq \overline{c o}\left(T\left(K_{1}\right)\right) .
$$

Similarly, $T\left(\overline{c o}\left(T\left(K_{1}\right)\right)\right) \subseteq \overline{c o}\left(T\left(K_{2}\right)\right)$, that is, $T$ is cyclic on $\overline{c o}\left(T\left(K_{2}\right)\right) \cup \overline{c o}\left(T\left(K_{1}\right)\right)$. The maximality of $\left(K_{1}, K_{2}\right)$ finishes the proof.

Theorem 2. Let $(A, B)$ be a nonempty, bounded, closed, and convex pair in a convex dualistic partial metric space $(X, \mathcal{D}, W)$ such that $\mathcal{D}$ is continuous and $\mathcal{D}(x, x) \leq 0$ for all $x \in A \cup B$. Let $\left(K_{1}, K_{2}\right) \subseteq(A, B)$ be a maximal pair with respect to being nonempty, closed and convex such that $T$ is cyclic on $K_{1} \cup K_{2}$. Suppose that $T: A \cup B \rightarrow A \cup B$ is a cyclic. Suppose that, for all $x \in K_{1}$ and $y \in K_{2}$,

$$
\mathcal{D}(T x, T y) \leq \Lambda:=\left\{k \delta\left(K_{1}, K_{2}\right)+(1-k) \operatorname{dist}(A, B)\right\}+\min \{\mathcal{D}(T x, T x), \mathcal{D}(T y, T y)\} .
$$

If $X$ has the property $(C)$, then $T$ has a best proximity pair.

Proof. Let $x \in K_{1}$ and $y \in K_{2}$; from the inequality fulfilled by the mapping $T$, we get $T y \in B(T x, \Lambda)$ and then

$$
T\left(K_{2}\right) \subseteq B(T x, \Lambda)
$$

thus,

$$
K_{1}=\overline{c o}\left(T\left(K_{2}\right)\right) \subseteq B(T x, \Lambda),
$$

which means,

$$
\mathcal{D}(T x, z) \leq \Lambda+\mathcal{D}(T x, T x), \text { for all } z \in K_{1}
$$


that is, $\delta_{T x}\left(K_{1}\right) \leq \Lambda+\mathcal{D}(T x, T x)$ and similarly we get $\delta_{T y}\left(K_{2}\right) \leq \Lambda+\mathcal{D}(T y, T y)$. Put

$$
L_{1}:=\left\{x \in K_{1}: \delta_{x}\left(K_{2}\right) \leq \Lambda+\mathcal{D}(x, x)\right\} \text { and } L_{2}:=\left\{y \in K_{2}: \delta_{y}\left(K_{1}\right) \leq \Lambda+\mathcal{D}(y, y)\right\} .
$$

Clearly, $\left(L_{1}, L_{2}\right)$ is a pair of nonempty, closed and convex subsets such that $T$ is cyclic on $L_{1} \cup L_{2}$. We take account of the maximilaty of $\left(K_{1}, K_{2}\right)$ to conclude that $L_{1}=K_{1}$ and $L_{2}=K_{2}$-from which we get

$$
\delta_{x}\left(K_{2}\right) \leq r \delta\left(K_{1}, K_{2}\right)+(1-r) \operatorname{dist}(A, B)+\mathcal{D}(x, x) \text { for all } x \in K_{1} .
$$

Hence,

$$
\delta\left(K_{1}, K_{2}\right)=\operatorname{dist}(A, B) .
$$

Consequently,

$$
\operatorname{dist}(A, B) \leq \mathcal{D}(p, T p), \mathcal{D}(T q, q) \leq \delta\left(K_{1}, K_{2}\right)=\operatorname{dist}(A, B), \text { for all }(p, q) \in K_{1} \times K_{2} .
$$

In addition, that is the desired result.

The next corollary follows immediately.

Corollary 2 ([1]). Let $(A, B)$ be a nonempty, bounded, closed, and convex pair in a convex metric space $(X, d, W)$. Suppose that $T: A \cup B \rightarrow A \cup B$ is a generalized cyclic contraction. If $X$ has the $(C)$ property, then $T$ has a best proximity pair.

\section{Tricyclic Mappings in Convex Extended Partial $S_{b}$ Metric Spaces}

Lately, extended partial $S_{b}$-metric spaces were introduced as comes

Definition 10 ([7]). Let $X$ be a nonempty subset and let $\theta: X^{3} \longrightarrow[1, \infty)$. If a mapping $S_{\theta}: X^{3} \longrightarrow[0, \infty)$ satisfies 1. $x=y=z$ if and only if $S_{\theta}(x, y, z)=S_{\theta}(x, x, x)=S_{\theta}(y, y, y)=S_{\theta}(z, z, z)$,

2. $S_{\theta}(x, x, x) \leq S_{\theta}(x, y, z)$,

3. $S_{\theta}(x, y, z) \leq \theta(x, y, z)\left[S_{\theta}(x, x, t)+S_{\theta}(y, y, t)+S_{\theta}(z, z, t)\right]$, for all $x, y, z, t \in X$. Then, $\left(X, S_{\theta}\right)$ is called an extended partial $S_{b}$-metric space.

Next, we introduce the notion of convexity in extended partial $S_{b}$-metric spaces.

Definition 11. Let $\left(X, S_{\theta}\right)$ be an extended partial $S_{b}$-metric space. A mapping $W: X \times X \times[0,1] \longrightarrow X$ is said to be a convex structure on $X$ if, for each $(x, y) \in X \times X$ and $\lambda \in[0,1]$,

$$
S_{\theta}(u, v, W(x, y, \lambda)) \leq \lambda S_{\theta}(u, v, x)+(1-\lambda) S_{\theta}(u, v, y) \text { for all } u, v \in X .
$$

In addition, $\left(X, S_{\theta}, W\right)$ is said to be a convex extended partial $S_{b}$-metric space.

It is easy to see that every convex metric space in the sense of [15] is a convex extended partial $S_{b}$-metric space. Now, we present a yet stronger version of convexity.

Definition 12. Retaining the same notations as in the previous definition, $W$ is said to be a double convex structure on $X$ if it is a convex structure and if, for each $\left(x_{1}, y_{1}\right),\left(x_{2}, y_{2}\right) \in X \times X, \lambda \in[0,1]$ and $u \in X$,

$$
S_{\theta}\left(u, W\left(x_{1}, y_{1}, \lambda\right), W\left(x_{2}, y_{2}, \lambda\right)\right) \leq \lambda S_{\theta}\left(u, x_{1}, x_{2}\right)+(1-\lambda) S_{\theta}\left(u, y_{1}, y_{2}\right) .
$$

Example 2. Let $(X,\|\|$.$) be a normed linear space and S_{\theta}: X^{3} \longrightarrow[0, \infty)$ be defined as $S_{\theta}(x, y, z)=$ $\|x-y\|+\|y-z\|+\|z-x\|$. Then, $\left(X, S_{\theta}\right)$ is an extended partial $S_{b}$-metric space and the mapping $W$ : 
$X \times X \times[0,1] \longrightarrow X$ defined by $W(x, y, \lambda)=\lambda x+(1-\lambda) y$ is a convex structure on $X$. Moreover, $W$ is a double convex structure. Indeed, fix $\left(x_{1}, y_{1}\right),\left(x_{2}, y_{2}\right) \in X \times X, \lambda \in[0,1]$ and $u \in X$, we have

$$
\begin{aligned}
S_{\theta}\left(u, W\left(x_{1}, y_{1}, \lambda\right), W\left(x_{2}, y_{2}, \lambda\right)\right)= & \left\|u-\lambda x_{1}-(1-\lambda) y_{1}\right\| \\
& +\left\|u-\lambda x_{2}-(1-\lambda) y_{2}\right\| \\
& +\left\|\lambda x_{1}+(1-\lambda) y_{1}-\lambda x_{2}-(1-\lambda) y_{2}\right\| \\
\leq & \lambda\left\|u-x_{1}\right\|+(1-\lambda)\left\|u-y_{1}\right\| \\
& +\lambda\left\|u-x_{2}\right\|+(1-\lambda)\left\|u-y_{2}\right\| \\
& +\lambda\left\|x_{1}-x_{2}\right\|+(1-\lambda)\left\|y_{1}-y_{2}\right\| \\
= & \lambda S_{\theta}\left(u, x_{1}, x_{2}\right)+(1-\lambda) S_{\theta}\left(u, y_{1}, y_{2}\right) .
\end{aligned}
$$

From now on, $\left(X, S_{\theta}, W\right)$ will denote a convex extended partial $S_{b}$-metric space.

Definition 13. A subset $K$ of $X$ is said to be convex if $W(x, y, \lambda) \in K$ for all $x, y \in X$ and $\lambda \in[0,1]$.

Definition 14. For all $x, y \in X$ and $\varepsilon>0$, the ball of foci $x$ and $y$, and of ray $\varepsilon$ is given by

$$
B(x, y, \varepsilon)=\left\{z \in X: S_{\theta}(x, y, z) \leq \varepsilon\right\} .
$$

The following propositions follow from the aforementioned definitions immediately.

Proposition 3 ([21]). Let $\left\{K_{\alpha}\right\}_{\alpha}$ be a family of convex subsets of the convex extended partial $S_{b}$-metric space $X$, then $\cap K_{\alpha}$ is a convex subset of $X$ as well.

Proposition 4. The balls $B(x, y, \varepsilon)$ are convex subsets of $X$. Moreover, they are closed subsets whenever $S_{\theta}$ is a continuous mapping.

Proof. Let $a, b \in B(x, y, \varepsilon)$ and $\lambda \in[0,1]$.

$$
\begin{aligned}
S_{\theta}(x, y, W(a, b, \lambda)) & \leq \lambda S_{\theta}(x, y, a)+(1-\lambda) S_{\theta}(x, y, b) \\
& \leq \lambda \varepsilon+(1-\lambda) \varepsilon=\varepsilon .
\end{aligned}
$$

Furthermore, $B(x, y, \varepsilon)=T^{-1}([0, \varepsilon])$ where $T(z)=S_{\theta}(x, y, z)$ for all $z \in X$. The balls $B(x, y, \varepsilon)$ are closed subsets if $S_{\theta}$ is continuous.

Before getting to our main result of this section, we fix some notations. Let $A, B$ and $C$ be nonempty subsets of $\left(X, S_{\theta}, W\right)$ :

$$
\begin{aligned}
\operatorname{dist}(A, B, C) & =\inf \left\{S_{\theta}(x, y, z): x \in A, y \in B \text { and } z \in C\right\} \\
\delta(A, B, C) & =\sup \left\{S_{\theta}(x, y, z): x \in A, y \in B \text { and } z \in C\right\} \\
\delta_{(x, y)}(C) & =\sup \left\{S_{\theta}(x, y, z): z \in C\right\} \text { for all } x \in A \text { and } y \in B
\end{aligned}
$$

Take note that extended partial $S_{b}$-metric spaces are, sort of, three-dimensional metric spaces and, since a tricyclic mapping is defined on the union of three subsets, the definition of a best proximity point for a tricylic mapping is naturally given by:

Definition 15. Let $T: A \cup B \cup C \longrightarrow A \cup B \cup C$ be a tricyclic mapping where $A, B$ and $C$ are nonempty subsets of $\left(X, S_{\theta}\right)$. A point $x \in A \cup B \cup C$ is said to be a best proximity point for $T$ provided that

$$
S_{\theta}\left(x, T x, T^{2} x\right)=\operatorname{dist}(A, B, C) .
$$


Lemma 2. Let $(A, B, C)$ be a nonempty, bounded, closed, and convex triad in $X$. Suppose that $T: A \cup B \cup$ $C \longrightarrow A \cup B \cup C$ is a tricyclic mapping. If $X$ has the property $(C)$, then there exists a triad $\left(K_{1}, K_{2}, K_{3}\right) \subseteq$ $(A, B)$ which is maximal with respect to being nonempty, closed and convex such that $T$ is tricyclic on $K_{1} \cup K_{2}$. Furthermore,

$$
\overline{c o}\left(T\left(K_{1}\right)\right)=K_{2}, \overline{c o}\left(T\left(K_{2}\right)\right)=K_{3} \text { and } \overline{c o}\left(T\left(K_{3}\right)\right)=K_{1} .
$$

Proof. Let $\Gamma$ denote the set of all nonempty, closed, and convex triads $(I, J, H) \subseteq(A, B, C)$ such that $T$ is tricyclic on $I \cup J \cup H$. Note that $\Gamma$ is partially ordered by reverse inclusion, that is,

$$
\left(I_{1}, J_{1}, H_{1}\right) \leq\left(I_{2}, J_{2}, H_{2}\right) \Longleftrightarrow\left(I_{2}, J_{2}, H_{2}\right) \subseteq\left(I_{1}, J_{1}, H_{1}\right)
$$

Let $\left\{\left(I_{\alpha}, J_{\alpha}, H_{\alpha}\right)\right\}_{\alpha}$ be an increasing chain of $\Gamma$. Since $X$ has the property $(C)$ and from the fact that every intersection of convex subsets is a convex subset, $\left(\cap I_{\alpha}, \cap J_{\alpha}, \cap H_{\alpha}\right)$ is a nonempty, closed and convex triad. In addition, the maximal triad $\left(K_{1}, K_{2}, K_{3}\right)$ is obtained as Zorn's Lemma states. Now, the triad $\left(\overline{c o}\left(T\left(K_{3}\right)\right), \overline{c o}\left(T\left(K_{1}\right)\right), \overline{c o}\left(T\left(K_{2}\right)\right)\right) \subseteq\left(K_{1}, K_{2}, K_{3}\right)$ is nonempty, closed and convex. We also have

$$
T\left(\overline{c O}\left(T\left(K_{3}\right)\right)\right) \subseteq T\left(K_{1}\right) \subseteq \overline{c o}\left(T\left(K_{1}\right)\right)
$$

Similarly, we see that $T$ is tricyclic on $\overline{c o}\left(T\left(K_{3}\right)\right) \cup \overline{c o}\left(T\left(K_{1}\right)\right) \cup \overline{c o}\left(T\left(K_{3}\right)\right)$. The desired result follows from the maximality of $\left(K_{1}, K_{2}, K_{3}\right)$.

Theorem 3. Let $(A, B, C)$ be a nonempty, bounded, closed, and convex triad in $X$ such that $S_{\theta}$ is continuous and $W$ is a double convex strusture. Let $\left(K_{1}, K_{2}, K_{3}\right) \subseteq(A, B, C)$ be a maximal triad with respect to being nonempty, closed and convex such that $T$ is tricyclic on $K_{1} \cup K_{2} \cup K_{3}$. Suppose that $T: A \cup B \cup C \longrightarrow A \cup B \cup C$ is a tricyclic mapping such that

$$
S_{\theta}(T x, T y, T z) \leq \Lambda:=k \delta\left(K_{1}, K_{2}, K_{3}\right)+(1-k) \operatorname{dist}(A, B, C)
$$

for all $(x, y, z) \in K_{1} \times K_{2} \times K_{3}$. If $X$ has the property $(C)$ then $T$ has a best proximity triad.

Proof. Let $x \in K_{1}, y \in K_{2}$; the inequality satisfied by the mapping $T$ implies that $T z \in B(T x, T y, \Lambda)$ for all $z \in K_{3}$ and that means

$$
T\left(K_{3}\right) \subseteq B(T x, T y, \Lambda)
$$

Since $S_{\theta}$ is continuous, $B(T x, T y, \Lambda)$ is closed. Thus,

$$
K_{1}=\overline{c o}\left(T\left(K_{3}\right)\right) \subseteq B(T x, T y, \Lambda)
$$

Thus,

$$
\delta_{(T x, T y)}\left(K_{1}\right) \leq \Lambda
$$

Put

$$
\begin{aligned}
& L_{1}:=\left\{(x, y) \in K_{1} \times K_{2}: \delta_{(x, y)}\left(K_{3}\right) \leq \Lambda\right\}, \\
& L_{2}:=\left\{(y, z) \in K_{2} \times K_{3}: \delta_{(y, z)}\left(K_{1}\right) \leq \Lambda\right\}, \\
& L_{3}:=\left\{(z, x) \in K_{3} \times K_{1}: \delta_{(z, x)}\left(K_{2}\right) \leq \Lambda\right\} .
\end{aligned}
$$

Clearly, $\left(L_{1}, L_{2}, L_{3}\right)$ is a triad of nonempty, closed and convex subsets. Define

$$
\begin{gathered}
\widetilde{T}:(A \times B) \cup(B \times C) \cup(C \times A) \longrightarrow(A \times B) \cup(B \times C) \cup(C \times A) \\
(x, y) \longmapsto \widetilde{T}(x, y)=(T x, T y)
\end{gathered}
$$


Since $T$ is tricyclic on $A \cup B \cup C, \widetilde{T}$ is tricyclic on $(A \times B) \cup(B \times C) \cup(C \times A)$. For all $(x, y) \in K_{1} \times K_{2}$, $\widetilde{T}(x, y)=(T x, T y) \in L_{2}$, then $\widetilde{T}\left(K_{1} \times K_{2}\right) \subseteq L_{2}$. Thus, $\widetilde{T}$ is tricyclic on $L_{1} \cup L_{2} \cup L_{3}$. Furthermore, $\left(K_{1} \times K_{2}, K_{2} \times K_{3}, K_{3} \times K_{1}\right)$ is maximal in

$$
\widetilde{\Gamma}=\left\{\begin{array}{l}
((I \times J),(J \times H),(H \times I)) \subseteq((A \times B),(B \times C),(C \times A)) / \\
(I \times J),(J \times H) \text { and }(H \times I) \text { are non-empty, bounded, closed } \\
\text { and convex with } \widetilde{T} \text { is tricyclic on }(I \times J) \cup(J \times H) \cup(H \times I)
\end{array}\right\},
$$

which is partially ordered by

$$
\begin{gathered}
\left(\left(I_{1} \times J_{1}\right),\left(J_{1} \times H_{1}\right),\left(H_{1} \times I_{1}\right)\right) \widetilde{\leq}\left(\left(I_{2} \times J_{2}\right),\left(J_{2} \times H_{2}\right),\left(H_{2} \times I_{2}\right)\right) \Longleftrightarrow \\
\left(\left(I_{2} \times J_{2}\right),\left(J_{2} \times H_{2}\right),\left(H_{2} \times I_{2}\right)\right) \subseteq\left(\left(I_{1} \times J_{1}\right),\left(J_{1} \times H_{1}\right),\left(H_{1} \times I_{1}\right)\right) .
\end{gathered}
$$

Therefore,

$$
L_{1}=K_{1} \times K_{2}, L_{2}=K_{2} \times K_{3} \text { and } L_{3}=K_{3} \times K_{1} .
$$

Consequently, for all $(x, y) \in K_{1} \times K_{2}$,

$$
\delta_{(x, y)}\left(K_{3}\right)-k \delta\left(K_{1}, K_{2}, K_{3}\right) \leq(1-k) \operatorname{dist}(A, B, C) .
$$

That is,

$$
\delta\left(K_{1}, K_{2}, K_{3}\right) \leq \operatorname{dist}(A, B, C) .
$$

Now, for all $(p, q, r) \in K_{1} \times K_{2} \times K_{3}$, we get

$$
\begin{aligned}
\operatorname{dist}(A, B, C) & \leq S_{\theta}\left(p, T p, T^{2} p\right), S_{\theta}\left(q, T q, T^{2} q\right), S_{\theta}\left(r, T r, T^{2} r\right) \\
& \leq \delta\left(K_{1}, K_{2}, K_{3}\right) \leq \operatorname{dist}(A, B, C) .
\end{aligned}
$$

In addition, this is a best proximity triad.

As a particular case of the previous theorem, we get the following result.

Corollary 3 ([12]). Let $A, B$ and $C$ be nonempty, closed, bounded and convex subsets of reflexive Banach space $X$, let $T: A \cup B \cup C \longrightarrow A \cup B \cup C$ be a tricyclic contraction map i.e.,

$$
D(T x, T y, T z) \leq k D(x, y, z)+(1-k) \operatorname{dist}(A, B, C) \text { for all }(x, y, z) \in A \times B \times C,
$$

where $D(x, y, z)=\|x-y\|+\|y-z\|+\|z-x\|$. Then, T has a best proximity triad.

\section{Conclusions}

In this work, we have provided two best approximation result for cyclic mappings in thesetting of dualistic partial and convex, metric spaces. Next, we have provided best proximity point existence result for a new class of tricyclic mappings. Our three results extend and improve some results in the literature.

Author Contributions: Conceptualization, T.S.; Supervision, A.B. and M.A.; Validation, M.A.; Writing-original draft, T.S. and A.B.

Funding: This research received no external funding.

Acknowledgments: Research was supported by a National Centre of Scientific and Technological Research grant. The authors would like to express their gratitude to the editor and the anonymous referees for their constructive comments and suggestions, which have improved the quality of the manuscript.

Conflicts of Interest: The authors declare no conflict of interest. 


\section{References}

1. Gabeleh, M.; Shahzad, N. Some new results on cyclic relatively nonexpansive mappings in convex metric spaces. J. Inequal. Appl. 2014, 2014, 350. [CrossRef]

2. Aydi, H.; Karapinar, E.; Erhan, I.M. Best proximity points of generalized almost $\Psi$-Geraghty contractive non-self-mappings. Fixed Point Theory Appl. 2014, 2014, 32. [CrossRef]

3. Kumam, P.; Aydi, H.; Karapınar, E.; Sintunavarat, W. Best proximity points and extension of MizoguchiTakahashi's fixed point theorems. Fixed Point Theory Appl. 2013, 2013, 242. [CrossRef]

4. Pitea, A. Best proximity results on dualistic partial metric spaces. Symmetry 2019, 11, 306. [CrossRef]

5. Eldered, A.A; Veeramani, P. Convergence and existence for best proximity points. J. Math. Anal. Appl. 2006, 323, 1001-1006. [CrossRef]

6. Sankar Raj, V.; Veeramani, P. Best proximity pair theorems for relatively nonexpansive mappings. Appl. Gen. Topol. 2009, 10, 21-28. [CrossRef]

7. Eldred, A.; Kirk, W.A.; Veeramani, P. Proximal normal structure and relatively nonexpansive mappings. Stud. Math. 2005, 171, 283-293. [CrossRef]

8. Sankar Raj, V. A best proximity point theorem for weakly contractive non-self-mappings. Nonlinear Anal. Theory Methods Appl. 2011, 74, 4804-4808. [CrossRef]

9. Abkar, A.; Gabaleh, M. A note on some best proximity point theorems proved under P-property. Abstr. Appl. Anal. 2013, 2013, 189567. [CrossRef]

10. Almeida, A.; Karapinar, E.; Sadrangani, K. A note on best proximity point theorems under weak P-property. Abstr. Appl. Anal. 2014, 2014, 716825. [CrossRef]

11. Karapinar, E.; Khojasteh, F. An approach to best proximity points results via simulation functions. J. Fixed Point Theory Appl. 2017, 19, 1983-1995. [CrossRef]

12. Sabar, T.; Aamri, M.; Bassou, A. Best proximity point of tricyclic contractions. Adv. Fixed Point Theory 2017, 7, 512-523.

13. Mukheimer, A. Extended partial Sb-metric spaces. Axioms 2018, 7, 87. [CrossRef]

14. O'Neill, S.J. Partial metric, valuations and domain theory. Ann. N. Y. Acad. Sci. 1996, 806, 304-315. [CrossRef]

15. Arshad, M.; Nazam, M.; Beg, I. Fixed point theorems in ordered dualistic partial metric spaces. Korean J. Math. 2016, 24, 169-179. [CrossRef]

16. Gabeleh, M.; Otafudu, O.O. Generalized pointwise noncyclic relatively nonexpansive mappings in strictly convex Banach spaces. J. Nonlinear Convex Anal. 2016, 17, 1117-1128.

17. Gabeleh, M.; Otafudu, O.O. Markov-Kakutani's theorem for best proximity pairs in Hadamard spaces. Indag. Math. 2017, 28, 680-693. [CrossRef]

18. Diaz, J.B.; Metcalf, F.T. On the structure of the set of subsequential limit points of successive approximations. Bull. Am. Math. Soc. 1967, 73, 51-59. [CrossRef]

19. Espínola, R.; Kosuru, G.S.R.; Veeramani, P. Pythagorean Property and Best-Proximity Point Theorems. J. Optim. Theory Appl. 2015, 164, 534-550. [CrossRef]

20. Kumam, P.; Mongkolkeha, C. Global optimization for quasi-noncyclic relatively nonexpansive mappings with application to analytic complex functions. Mathematics 2019, 7, 46. [CrossRef]

21. Takahashi, W. A convexity in metric space and nonexpansive mappings. Kodai Math. Semin. Rep. 1970, 22, 142-149. [CrossRef]

22. Shimizu, T.; Takahashi, W. Fixed points of multivalued mappings in certain convex metric spaces. Topol. Methods Nonlinear Anal. 1996, 8, 197-203. [CrossRef]

(c) 2019 by the authors. Licensee MDPI, Basel, Switzerland. This article is an open access article distributed under the terms and conditions of the Creative Commons Attribution (CC BY) license (http:/ / creativecommons.org/licenses/by/4.0/). 